DOI 10.12957/demetra.2014.4851

\title{
Obesidade abdominal em crianças escolares: prevalência e fatores associados
}

\section{Abdominal obesity in school children: prevalence and associated factors}

\author{
Natália Basso Ferrazzi ${ }^{1}$ \\ Ana Caroline Branco ${ }^{\top}$ \\ Doroteia Aparecida Höfelmann' \\ ${ }^{1}$ Curso de Nutrição da Universidade do Vale do \\ Itajaí. Itajaí, SC, Brasil. \\ Fonte financiadora: 0 trabalho realizado recebeu \\ apoio financeiro da Bolsa de Pesquisa do Artigo \\ 170, da Universidade do Vale do Itajaí, e Governo \\ do Estado de Santa Catarina. \\ Correspondência/ Correspondence \\ Doroteia Aparecida Höfelmann \\ Universidade do Vale do Itajá - UNIVALI \\ Rua Uruguai, 458. Centro, Itajaí/Santa Catarina, \\ Brasil. CEP: 88302-202. \\ E-mail:doroaph@yahoo.com.br
}

\section{Resumo}

Estudo transversal objetivou estimar a prevalência de obesidade abdominal e verificar sua associação com variáveis demográficas, socioeconômicas, antropométricas, imagem corporal e comportamentos a ela relacionados em crianças e seus responsáveis. Foram sorteados alunos $(\mathrm{n}=717)$ de 22 escolas municipais do $4^{\circ}$ e $5^{\circ}$ anos de Itajaí, Santa Catarina. As variáveis foram coletadas por meio de questionário aplicado às crianças e aos responsáveis. Coletaram-se medidas de massa corporal, estatura e circunferência da cintura (indicador de obesidade abdominal). Razões de chance (RC) brutas, ajustadas e respectivos intervalos de confiança de 95\% (IC 95\%) foram calculados por meio de regressão logística não condicional. As análises foram ajustadas para o efeito do delineamento. Participaram da pesquisa 602 crianças $(81,7 \%)$, com idade média de 9,9 anos. A prevalência de obesidade abdominal foi de 11,3\% (IC 95\%: 8,714,0\%). Variáveis do responsável como renda média (RC: 3,7; IC 95\%: 1,4-10,1) ou alta (RC: 2,6; IC 95\%: 1,0-6,7), e presença de excesso de massa corporal (RC: 4,9; IC 95\%: 1,2-20,2) estiveram associadas à maior prevalência de obesidade abdominal entre os escolares. A realização de dietas para reduzir massa corporal (RC: 3,9; IC 95\%: 1,7-9,1) e a avaliação da saúde da criança pelos pais como regular ou ruim (RC 3,6 IC 95\%, 1,0-13,2) estiveram associadas ao desfecho. Medidas de intervenção destinadas a reduzir a prevalência de obesidade abdominal entre as crianças investigadas devem incluir os pais.

Palavras-chave: Obesidade Pediátrica, Saúde da Criança. Obesidade abdominal. Circunferência abdominal. Pais. Sobrepeso. 


\section{Abstract}

Cross-sectional study aimed to estimate the prevalence of abdominal obesity and its association with demographic, socioeconomic, anthropometric, body image and behaviors related to its variables among infants and their caregivers. Were randomly selected students $(\mathrm{n}=717)$ from 22 municipal schools in the 4th and 5th year of Itajai city, Santa Catarina state, Brazil. Variables were collected through a questionnaire applied to children and caregivers. We collected measures of weight, height and waist circumference (indicator of abdominal obesity). Crude and adjusted odds ratios (OR) and 95\% confidence intervals (95\% CI) were calculated through unconditional logistic regression. All analyses were adjusted for the design effect. Participants were 602 children (81.7\%), with age average of 9.9 years. Prevalence of abdominal obesity was $11.3 \%$ (95\% CI: 8.7 to $14.0 \%)$. Caregivers variables as middle (OR: 3.7, 95\% CI: 1.4 to 10.1) or high income (OR: 2.6, 95\% CI 1.0 to 6.7 ), and overweight (OR: $4.9,95 \%$ CI: 1.2 to 20.2 ) were associated with higher prevalence of abdominal obesity among school children. Diets to lose weight (OR: 3.9, 95\% CI 1.7 to 9.1), and assessment of child health by parents as fair or poor (OR 3.6 95\% CI, 10.0 to 13, 2) were associated with the outcome. Intervention measures to reduce the prevalence of obesity among children investigated should include parents.

Key words: Pediatric Obesity, Child Health. Abdominal obesity. Waist circumference. Parents. Overweight.

\section{Introdução}

Nas últimas décadas, houve rápido e significativo aumento na prevalência mundial de obesidade, especialmente durante a infância e a adolescência, tomando proporções de uma epidemia mundial. A obesidade na infância ou adolescência pode perdurar até a fase adulta, implicando aumento do risco de problemas vasculares e metabólicos. ${ }^{1}$

Diferentes medidas antropométricas têm sido destacadas para identificar a obesidade. O índice de massa corporal (IMC) reflete o excesso de gordura corporal total e tem sido o método antropométrico mais utilizado para avaliar o estado nutricional e diagnosticar sobrepeso corporal e obesidade. Entretanto, tem-se observado uma preocupação com o tipo de depósito de distribuição de gordura, uma vez que esta se relaciona com o prognóstico de risco para problemas de saúde. $\mathrm{Na}$ adiposidade central, a distribuição de tecido adiposo se dá preferencialmente no nível do 
tronco, com deposição aumentada em região intra-abdominal. ${ }^{2,3}$ Os riscos associados à obesidade abdominal a tornam um dos critérios diagnósticos da síndrome metabólica também em crianças. ${ }^{4,5}$

Crianças com obesidade abdominal podem apresentar maior prevalência de fatores de risco para doenças cardiovasculares, incluindo concentrações maiores de triglicerídeos, insulina, leptina, pressão arterial sistólica e diastólica, além de HDL-C (high density lipoprotein) reduzido. ${ }^{6}$ Além disso, em estudo longitudinal os autores verificaram que mais de metade das crianças com obesidade abdominal aos sete anos de idade permaneceram na mesma categoria de risco na adolescência, enquanto apenas menos de $2 \%$ passaram para categorias de menor risco aos 15 anos de idade. ${ }^{7}$

A maior parte dos estudos que analisou os fatores associados à obesidade abdominal em crianças concentrou-se na descrição de variáveis bioquímicas e/ou antropométricas. ${ }^{2,3,8}$ Variáveis maternas, como o excesso de massa corporal, têm sido associadas à deposição de gordura visceral em crianças. ${ }^{9}$

Estudos publicados no Brasil que analisaram os fatores associados à obesidade abdominal em crianças não puderam ser identificados pelos autores. A maior parte dos estudos incluiu adolescentes. ${ }^{6,10}$ Desta forma, ao se considerar a escassez de estudos, bem como os impactos negativos da obesidade abdominal na população infantil e sua significativa probabilidade de continuidade na vida adulta, estimar a prevalência de obesidade abdominal e investigar sua associação com variáveis da família e da criança representa estratégia importante para identificar os grupos em situação de maior risco.

Deste modo, o presente estudo objetivou estimar a prevalência de obesidade abdominal e verificar sua associação com variáveis demográficas, socioeconômicas, antropométricas, imagem corporal e comportamentos a ela relacionados em crianças e do 4o e $5^{\circ}$ anos de escolas municipais de Itajaí, estado de Santa Catarina, e de seus responsáveis.

\section{Métodos}

Este artigo está inserido em projeto que avaliou a prevalência de insatisfação corporal e fatores associados em crianças de Itajaí-SC. Trata-se de estudo transversal realizado com escolares da rede municipal de Itajaí, matriculados no $4^{\mathrm{o}}$ e $5^{\mathrm{o}}$ anos do ensino fundamental, residentes no perímetro urbano e rural.

A cidade de Itajaí localiza-se no Vale do Itajaí, a 91 km da capital de Santa Catarina, tem como principais atividades econômicas o porto mercante e a pesca, e uma população de 183.388 pessoas. No ano de 2000, foi apontado Índice de Desenvolvimento Humano de 0,825 no município. ${ }^{11}$

O município apresentava 39 escolas municipais, com 4.587 alunos matriculados no $4^{\underline{o}}$ e $5^{\underline{o}}$ anos. A amostragem foi em duplo estágio. A listagem das escolas foi fornecida pela Secretaria de Educação de Itajaí. A partir desta lista, foram sorteadas aleatoriamente 22 escolas dentre as 
existentes para participar da pesquisa, número considerado adequado para o porte do município. ${ }^{12}$ Em cada escola sorteada, foi obtida uma listagem dos alunos matriculados para se proceder ao sorteio dos alunos para a coleta de dados.

No cálculo de amostra então realizado por meio do aplicativo Epi Info 6.04 (EUA - Center for Control of Diseases - CDC), optou-se por considerar uma prevalência esperada de $50 \%$ para maximizar o tamanho amostral e permitir estudar outros desfechos de interesse com prevalências diversas. Foram considerados nível de confiança de $95 \%$ e margem de erro de $5 \%$. Como a amostragem foi realizada em duplo estágio, estimou-se o efeito de delineamento de 1,5 ( $\mathrm{n}=531$ ), ao resultado foi acrescido $20 \%$ para compensar perdas e/ou recusas e $15 \%$ para controlar fatores de confusão $(\mathrm{n}=717)$.

A prevalência de obesidade abdominal entre as crianças avaliadas foi de 11,3\%, sendo que dentre as crianças avaliadas, 547 possuíam o dado coletado para esta variável. Desta forma, o cálculo a posteriori permitiu precisão de três pontos percentuais, mantidos efeito do delineamento de 1,5 e nível de confiança de $95 \%$.

No segundo estágio foram selecionados aqueles a serem incluídos no estudo, dentre os matriculados nas instituições sorteadas: os escolares foram numerados e em seguida, procedeu-se à seleção sistemática através de uma fração da amostra $(\mathrm{k})$ obtida pela divisão do número total de escolares matriculados pelo tamanho da amostra.

A coleta de dados foi realizada por acadêmicas de Nutrição responsáveis pelo projeto, entre fevereiro a julho de 2010, e compreendeu a coleta de dados antropométricos e aplicação de questionários para a criança e para seu responsável. Foi solicitada a assinatura do Termo de Consentimento Livre e Esclarecido (TCLE) pelos responsáveis das crianças. Foram incluídos no estudo os escolares sorteados que apresentaram o TCLE devidamente assinado. Consideraramse perdas escolares que, após três visitas à escola, não estiveram presentes, e recusas, aqueles que não apresentaram o termo de consentimento assinado.

O questionário enviado aos responsáveis incluiu perguntas sobre idade, escolaridade (até 8 anos; 9 a 11 anos e 12 ou mais anos), renda familiar per capita em salários mínimos ( $\mathrm{R} \$ 510,00)$ dividida em tercis: baixa $(0,11$ a 0,47$)$, média $(0,48$ a 0,78$)$, alta $(0,79$ a 3,27$)$, cor da pele (branca; parda/ preta), hábito de realizar dietas para perda de massa corporal (sim; não), percepção dos genitores em relação à massa corporal infantil e a sua própria (abaixo; adequada e acima) e avaliação da saúde infantil (muito boa; boa e regular/ruim). A cor da pele do escolar foi classificada pelos pesquisadores de acordo com categorias propostas pelo Instituto Brasileiro de Geografia e Estatística (IBGE). ${ }^{13}$ Além disso, os responsáveis responderam à questão relacionada a sua satisfação com a própria massa corporal (não; sim), e à realização de tentativas para redução da massa corporal (não; sim). 
Aplicou-se aos escolares uma escala de imagem corporal - Children's Figure Rating Scale ${ }^{14}$, composta por nove silhuetas numeradas, com extremos de magreza e gordura, na qual deveriam selecionar a figura compatível com seu corpo atual e aquela desejada. ${ }^{14}$ Adicionalmente, eles responderam a perguntas com alternativas "sim" e "não", em relação a tentativa de emagrecer ou de engordar, realização de dietas e percepção da massa corporal.

Para a avaliação do estado nutricional, os escolares foram pesados em balança adulto digital da marca Kratos-Ca (São Paulo), com capacidade máxima de 150 kg, com o mínimo possível de roupa e sem calçado. A balança estava apoiada numa superfície plana, firme e lisa, afastada da parede. A criança ficou no centro da balança descalça, ereta, com os pés juntos, os braços estendidos ao longo do corpo e parada nessa posição. ${ }^{15}$

Após a pesagem, a estatura foi aferida com a utilização de estadiômetro compacto da marca Wiso (China), com escala de 0 a $200 \mathrm{~cm}$, precisão de $0,1 \mathrm{~cm}$. Os escolares foram medidos descalços, em pé, sobre superfície plana, de costas para o estadiômetro, com os pés paralelos e os tornozelos unidos. $^{15}$

A circunferência da cintura foi considerada o indicador de obesidade abdominal. Ela foi medida com o auxilio de uma fita métrica intextensível no perímetro mais estreito, entre a última costela e a crista ilíaca das crianças, sem comprimir os tecidos, e foi classificada segundo os pontos de corte proposto por Taylor et al..$^{16}$

Calculou-se o IMC, dividindo a massa corporal pela estatura ao quadrado, cujo resultado é dado $\mathrm{em} \mathrm{kg} / \mathrm{m}^{2}$. O estado nutricional foi determinado por meio do critério estabelecido pela Organização Mundial da Saúde. ${ }^{17}$ Para as análises, os casos de baixo-peso e risco de baixo-peso corporal foram agrupados, bem como os casos de sobrepeso e obesidade; os eutróficos permaneceram separados.

A massa corporal e a estatura dos pais foram autorreferidas, e a partir dessas informações foi calculado o IMC, classificado de acordo com as categorias da OMS. ${ }^{18}$ Para as análises, os casos de desnutrição e eutrofia foram agrupados, e os de sobrepeso e obesidade foram analisados separadamente.

A digitação dos dados foi efetuada no aplicativo Epidata, no qual foram criadas proteções para entrada de dados. Os questionários foram revisados e codificados pelos pesquisadores, e para garantir a qualidade da digitação, foram duplamente digitados e conferidos no programa EpiInfo.

Para descrever as variáveis quantitativas, calcularam-se as médias e os desvios-padrão, valores mínimos, máximos e medianos. As variáveis categóricas foram descritas por meio de suas frequências absolutas (n) e relativas (\%), e intervalos de confiança de 95\% (IC $95 \%$ ).

A associação entre as variáveis e a obesidade abdominal foi analisada por meio do teste do qui-quadrado de Pearson, com ajuste para delineamentos complexos. A prevalência de obesidade 
abdominal foi adicionalmente comparada entre as categorias das variáveis de exposição por meio das razões de chance (RC) e respectivos IC 95\%. A análise ajustada foi conduzida por meio da regressão logística não condicional, com ajuste para delineamentos complexos. Incluíram-se na análise as variáveis que mantiveram o nível de confiança de $25 \%$ na análise bivariada. A entrada das variáveis na análise ajustada foi realizada em seis níveis sucessivos e teve início pelas variáveis dos pais, seguindo modelo hierárquico ${ }^{19}$ (figura 1).

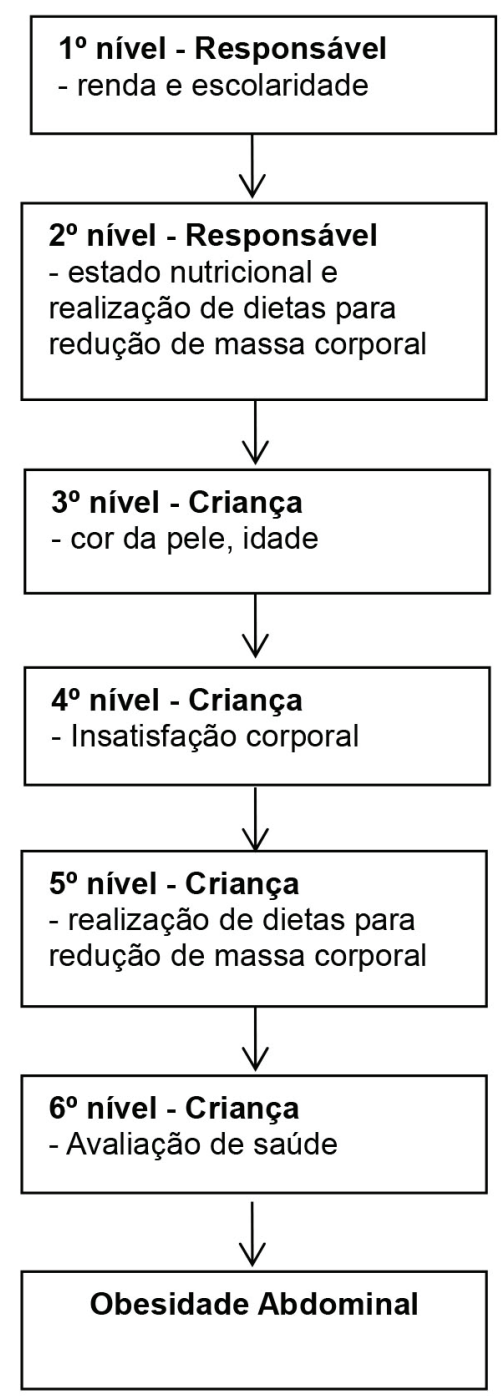

Figura 1: Modelo hierárquico de entrada de variáveis na análise multivariável. 
Consideraram-se significativas as associações quando valor de $\mathrm{p} \leq 0,05$. As análises foram realizadas por meio dos aplicativos Microsoft Excel, EpiInfo 6.04 e Stata SE9.

O projeto de pesquisa foi submetido e aprovado pelo Comitê de Ética em Pesquisa da instituição, sob protocolo número 373/09A.

\section{Resultados}

Participaram da pesquisa 602 crianças $(81,7 \%)$. A taxa de completude das questões variou de $67,4 \%$ para a variável renda, a $100 \%$ para aquelas coletadas no questionário respondido pelas crianças (tabela 2).

A maioria dos questionários foi respondida pelas mães $(82,0 \%)$, que autorreferiram cor de pele predominantemente branca $(69,8 \%)$ e até oito anos de estudo completos (47,5\%). Participaram do estudo mais meninas do que meninos (56,3\% versus 43,7\%). A média de idade foi de 9,9 anos e variou de 7,7 a 14,3 anos com desvio-padrão de 0,7 anos (tabela 1). Aproximadamente um terço das crianças apresentou excesso de massa corporal (31,6\%), segundo a tabela 2.

A prevalência de obesidade abdominal foi de 11,3\% (IC 95\% 8,7-14,0\%). Na análise bruta, as variáveis do responsável "renda", "IMC", "percepção e satisfação com a própria massa corporal" estiveram associadas à maior prevalência de obesidade abdominal entre as crianças avaliadas. Dentre as variáveis infantis, "insatisfação corporal", "realização de tentativas para perder massa corporal”, "realização de dietas", "percepção da massa corporal como acima do ideal”, bem como "avaliação de saúde da criança pelo responsável como regular/ruim" foram observadas com maior frequência entre as crianças com obesidade abdominal (tabela 2). Todas as crianças classificadas com obesidade abdominal estavam com excesso de massa corporal (dados não apresentados).

Após análise ajustada, a associação entre maior renda dos pais e obesidade abdominal se manteve. Filhos de pais com renda média ou alta apresentaram chances 3,7 e 2,6 vezes maior, respectivamente, de ter esse desfecho. Quando o responsável pela criança tinha sobrepeso ou obesidade, a chance de a criança apresentar obesidade abdominal foi 3,9 e 4,9 maior, respectivamente. Crianças com obesidade abdominal estiveram mais insatisfeitas com sua imagem corporal (RC 11,9 IC 95\% 1,3-108,6) e também apresentaram chances quatro vezes maiores de referir ter realizado dieta para redução de massa corporal. Além disso, crianças com obesidade abdominal apresentaram chance 3,2 vezes maior de ter sua saúde classificada como regular ou ruim pelos pais (tabela 3 ). 
Tabela 1. Descrição das de crianças do 4oe 5oa anos de escolas municipais de Itajaí-SC, 2010.

\begin{tabular}{lccc}
\hline \multicolumn{1}{c}{ Variáveis } & Meninos & Meninas & Total \\
\hline & Média (Dp) & Média (Dp) & Média (Dp) \\
\hline Idade (anos) & $9,9(0,7)$ & $9,8(0,7)$ & $9,9(0,7)$ \\
Massa corporal $(\mathrm{Kg})$ & $34,4(8,6)$ & $33,8(8,6)$ & $34,1(8,6)$ \\
Estatura $(\mathrm{cm})$ & $138,1(7,4)$ & $136,8(7,6)$ & $137,3(7,5)$ \\
Circunferência abdominal $(\mathrm{cm})$ & $59,9(8,0)$ & $57,9(7,5)$ & $58,7(7,8)$ \\
IMC $\left(\mathrm{Kg} / \mathrm{m}^{2}\right)$ & $17,9(3,1)$ & $17,9(3,4)$ & $17,9(3,3)$ \\
\hline
\end{tabular}

IMC = Índice de Massa Corporal; Dp = Desvio-padrão.

Tabela 2. Descrição das variáveis infantis e dos responsáveis por crianças do 4 o e $5^{\underline{o}}$ anos de escolas municipais de Itajaí-SC, 2010.

\begin{tabular}{llll}
\hline Variáveis & $\mathrm{n}$ & $\%$ & IC $95 \%$ \\
\hline Responsável & & & \\
\hline Cor da pele $(\mathrm{n}=503)$ & 351 & 69,8 & $65,5-73,7$ \\
Branca & 139 & 27,7 & $22,2-34,2$ \\
Parda/preta & & & \\
Escolaridade (n=480) & 228 & 47,5 & $39,0-56,0$ \\
Até 8 anos & 192 & 40,0 & $33,0-47,0$ \\
9 a 11 anos & 60 & 12,5 & $9,0-16,0$ \\
12 ou mais anos & & & \\
Renda familiar per capita $(\mathrm{n}=406)$ & 139 & 34,2 & $29,6-38,9$ \\
$1^{o}$ tercil (baixa) & 132 & 32,5 & $27,9-37,1$ \\
$2^{\text {o }}$ tercil (média) & 135 & 33,3 & $28,6-37,9$ \\
3o tercil (alta) & & & \\
IMC (n=417) & 201 & 48,2 & $43,4-53,0$ \\
Desnutrição/Eutrofia & 137 & 32,9 & $28,3-37,4$ \\
Sobrepeso & 79 & 18,9 & $15,2-22,7$ \\
Obesidade & & & \\
\end{tabular}




\begin{tabular}{|c|c|c|c|}
\hline Variáveis & $\mathrm{n}$ & $\%$ & IC $95 \%$ \\
\hline \multicolumn{4}{|c|}{$\begin{array}{l}\text { Realização de dietas para redução de massa } \\
\text { corporal }(n=490)\end{array}$} \\
\hline Sim & 130 & 26,5 & $22,7-30,7$ \\
\hline Não & 360 & 73,5 & $69,3-77,3$ \\
\hline \multicolumn{4}{|c|}{ Percepção da massa corporal $(n=485)$} \\
\hline Abaixo & 27 & 5,6 & $3,8-8,1$ \\
\hline Adequado & 191 & 39,4 & $35,0-43,9$ \\
\hline Acima & 267 & 55,1 & $50,5-59,5$ \\
\hline \multicolumn{4}{|c|}{ Satisfação com a massa corporal $(n=485)$} \\
\hline Sim & 157 & 32,4 & $28,2-36,5$ \\
\hline Não & 328 & 67,6 & $63,5-71,8$ \\
\hline \multicolumn{4}{|l|}{ Infantis } \\
\hline \multicolumn{4}{|l|}{$\operatorname{Sexo}(n=602)$} \\
\hline Masculino & 263 & 43,7 & $39,7-47,8$ \\
\hline Feminino & 339 & 56,3 & $52,2-60,3$ \\
\hline \multicolumn{4}{|c|}{ Cor da pele $(n=597)$} \\
\hline Branca & 433 & 71,9 & $68,1-75,4$ \\
\hline Parda & 95 & 15,8 & $13,0-19,0$ \\
\hline Preta/negra & 69 & 11,5 & $9,1-14,3$ \\
\hline \multicolumn{4}{|c|}{ Faixa etária $(n=602)$} \\
\hline Até 10 anos & 380 & 63,1 & $59,3-67,0$ \\
\hline 11 anos ou mais & 222 & 36,9 & $33,0-40,7$ \\
\hline \multicolumn{4}{|c|}{ Autopercepção da massa corporal $(\mathrm{n}=602)$} \\
\hline Abaixo & 128 & 21,3 & $16,7-25,8$ \\
\hline Adequado & 339 & 56,3 & $50,6-62,0$ \\
\hline Acima & 135 & 22,4 & $18,3-26,5$ \\
\hline
\end{tabular}




\begin{tabular}{|c|c|c|c|}
\hline Variáveis & $\mathrm{n}$ & $\%$ & IC $95 \%$ \\
\hline \multicolumn{4}{|c|}{ Insatisfação com imagem corporal $(n=602)$} \\
\hline Não & 139 & 23,1 & $19,7-26,5$ \\
\hline Sim & 463 & 76,9 & $73,5-80,3$ \\
\hline \multicolumn{4}{|c|}{ Percepção massa corporal pelos pais $(n=486)$} \\
\hline Abaixo & 64 & 13,2 & $10,4-16,6$ \\
\hline Adequado & 309 & 63,6 & $59,1-67,8$ \\
\hline Acima & 113 & 23,3 & $19,6-27,3$ \\
\hline \multicolumn{4}{|c|}{ Tentativa de engordar $(n=602)$} \\
\hline Sim & 137 & 22,8 & $19,5-26,4$ \\
\hline Não & 465 & 77,2 & $73,6-80,5$ \\
\hline \multicolumn{4}{|c|}{ Tentativa de emagrecer $(n=602)$} \\
\hline Sim & 283 & 47,0 & $43,0-51,1$ \\
\hline Não & 319 & 53,0 & $48,9-57,0$ \\
\hline \multicolumn{4}{|c|}{$\begin{array}{l}\text { Realização de dietas para redução de massa } \\
\text { corporal }(n=602)\end{array}$} \\
\hline Sim & 198 & 32,9 & $29,2-36,8$ \\
\hline Não & 404 & 67,1 & $29,2-36,8$ \\
\hline \multicolumn{4}{|c|}{ Avaliação da saúde pelos pais $(\mathrm{n}=492)$} \\
\hline Muito boa & 226 & 45,9 & $41,5-50,5$ \\
\hline Boa & 209 & 42,5 & $38,1-47,0$ \\
\hline Regular/ruim & 57 & 11,6 & $8,1-17,3$ \\
\hline \multicolumn{4}{|c|}{ Índice de massa corporal $(\mathrm{n}=602)$} \\
\hline Baixo & 56 & 9,3 & $7,2-12,0$ \\
\hline Adequado & 356 & 59,1 & $55,1-63,1$ \\
\hline Elevado & 190 & 31,6 & $27,9-35,5$ \\
\hline
\end{tabular}

Percentual (\%) e Intervalo de Confiança de 95\% (IC 95\%)

IMC - Índice de Massa Corporal 
Tabela 3. Razões de chance (RC) brutas e ajustadas, e respectivos intervalos de confiança de 95\% (IC 95\%) por meio da regressão logística não condicional, da associação entre obesidade abdominal e variáveis infantis e dos responsáveis por escolares de Itajaí-SC, 2010.

\begin{tabular}{|c|c|c|c|c|c|c|}
\hline Variáveis & $\mathrm{n}$ & $\%$ & $\begin{array}{l}\text { RC bruta } \\
\text { (IC 95\%) }\end{array}$ & $\mathrm{p}^{*}$ & $\begin{array}{l}\text { RC ajustada } \\
\text { (IC 95\%) }\end{array}$ & $\mathrm{p}^{* *}$ \\
\hline \multicolumn{7}{|l|}{ Responsável } \\
\hline Cor da pele & & & & 0,740 & & \\
\hline Branca & 40 & 12,7 & 1,0 & & & \\
\hline Preta/ Parda & 13 & 10,4 & $0,80(0,4-1,4)$ & & & \\
\hline Escolaridade & & & & 0,332 & & \\
\hline Até 8 anos & 25 & 12,1 & 1,0 & & & \\
\hline 9 a 11 anos & 18 & 10,8 & $0,9(0,6-1,3)$ & & & \\
\hline 12 ou mais anos & 9 & 16,4 & $1,4(0,6-3,0)$ & & & \\
\hline Renda familiar per capita & & & & 0,026 & & 0,026 \\
\hline $1^{\circ}$ tercil (baixa) & 7 & 5,5 & 1,0 & & 1,0 & \\
\hline $2^{\circ} \mathrm{a}$ tercil (média) & 20 & 17,7 & $3,7(1,4-10,1)$ & & $3,7(1,4-10,1)$ & \\
\hline $3^{\circ}$ tercil (alta) & 16 & 13,3 & $2,6(1,0-6,7)$ & & $2,6(1,0-6,7)$ & \\
\hline IMC & & & & 0,005 & & $\mathbf{0 , 0 1 6}$ \\
\hline Desnutrição/Eutrofia & 11 & 6,2 & 1,0 & & 1,0 & \\
\hline Sobrepeso corporal & 19 & 15,4 & $2,8(1,2-6,2)$ & & $3,9(1,5-10,0)$ & \\
\hline Obesidade & 17 & 23,6 & $4,7(1,6-13,3)$ & & $4,9(1,2-20,2)$ & \\
\hline $\begin{array}{l}\text { Realização de dietas para } \\
\text { redução da massa corporal }\end{array}$ & & & & 0,316 & & \\
\hline Não & 37 & 11,5 & 1,0 & & & \\
\hline Sim & 16 & 13,8 & $1,2(0,8-1,9)$ & & & \\
\hline Percepção da massa corporal & & & & 0,021 & & \\
\hline Abaixo & 0 & 0 & $\S$ & & & \\
\hline Adequado & 15 & 8,6 & $\S$ & & & \\
\hline Acima corporal & 38 & 16,1 & $\S$ & & & \\
\hline $\begin{array}{l}\text { Satisfação com a massa } \\
\text { corporal }\end{array}$ & & & & 0,021 & & \\
\hline Sim & 9 & 6,3 & 1,0 & & & \\
\hline Não & 44 & 15,2 & $2,7(1,2-6,2)$ & & & \\
\hline
\end{tabular}




\begin{tabular}{|c|c|c|c|c|c|c|}
\hline Variáveis & $\mathrm{n}$ & $\%$ & $\begin{array}{l}\text { RC bruta } \\
\text { (IC 95\%) }\end{array}$ & $\mathrm{p}^{*}$ & $\begin{array}{l}\text { RC ajustada } \\
\quad \text { (IC 95\%) }\end{array}$ & $\mathrm{p}^{* *}$ \\
\hline \multicolumn{7}{|l|}{ Infantis } \\
\hline Sexo & & & & 0,081 & & \\
\hline Masculino & 34 & 14,7 & 1,0 & & & \\
\hline Feminino & 28 & 8,9 & $0,6(0,3-1,1)$ & & & \\
\hline Cor da pele & & & & 0,920 & & \\
\hline Branca & 46 & 11,6 & 1,0 & & & \\
\hline Parda & 9 & 10,2 & $0,9(0,4-1,, 8)$ & & & \\
\hline Preta & 7 & 11,2 & $1,0(0,4-2,8)$ & & & \\
\hline Faixa etária & & & & 0,020 & & 0,015 \\
\hline Até 10 anos & 45 & 72,6 & 1,0 & & 1,0 & \\
\hline 11 anos ou mais & 17 & 27,4 & $0,6(0,4-0,9)$ & & $0,6(0,4-0,9)$ & \\
\hline \multicolumn{7}{|l|}{ corporal } \\
\hline Magro & 0 & 0 & $\S$ & & & \\
\hline Normal & 10 & 3,3 & 1,0 & & & \\
\hline Gordo & 52 & 41,9 & $\S$ & & & \\
\hline \multicolumn{4}{|c|}{ Percepção massa corporal pelos pais } & $<0,001$ & & \\
\hline Abaixo & 0 & 0 & 1,0 & & & \\
\hline Adequado & 3 & 1,1 & $15,2(5,8-39,5)$ & & & \\
\hline Acima & 49 & 52,1 & $\begin{array}{c}101,2(28,1- \\
364,2)\end{array}$ & & & \\
\hline Insatisfação corporal & & & & 0,002 & & 0,031 \\
\hline Não & 2 & 1,6 & 1,0 & & 1,0 & \\
\hline Sim & 60 & 14,3 & $10,4(2,0-55,0)$ & & $11,9(1,3-108,6)$ & \\
\hline Tentativa de emagrecer & & & & $<0,001$ & & \\
\hline Não & 7 & 2,4 & 1,0 & & & \\
\hline Sim & 55 & 21,5 & $11,1(5,4-22,7)$ & & & \\
\hline
\end{tabular}




\begin{tabular}{lcccccc}
\hline Variáveis & $\mathrm{n}$ & $\%$ & $\begin{array}{c}\text { RC bruta } \\
\text { (IC 95\%) }\end{array}$ & $\mathrm{p}^{*}$ & $\begin{array}{c}\text { RC ajustada } \\
\text { (IC 95\%) }\end{array}$ & $\mathrm{p}^{* * *}$ \\
\hline $\begin{array}{l}\text { Realização de dietas para } \\
\text { redução de massa corporal }\end{array}$ & 25 & 6,70 & 1,0 & 0,001 & & $\mathbf{0 , 0 0 3}$ \\
Não & 37 & 21,3 & $3,7(1,9-7,6)$ & & $3,9(1,7-9,09)$ & \\
Sim & & & & 0,012 & & $\mathbf{0 , 0 1 4}$ \\
Avaliação da saúde pelos pais & 16 & 7,9 & 1,0 & & 1,0 & \\
Muito boa & 27 & 14,2 & $1,9(1,0-3,7)$ & & $2,0(0,7-6,0)$ & \\
Boa & 10 & 21,3 & $3,2(1,4-6,8)$ & & $3,6(1,0-13,2)$ & \\
Regular/Ruim & & & & &
\end{tabular}

IMC = Índice de Massa Corporal

*Teste do Qui-quadrado de Pearson, com ajuste para efeito do delineamento.

** Teste de Wald com ajuste para efeito do delineamento.

$\S$ algumas informações apresentaram valor nulo, portanto RC não pode ser calculada.

\section{Discussão}

O presente estudo objetivou identificar a prevalência de obesidade abdominal e sua associação com fatores socioeconômicos, demográficos, antropométricos, percepções e atitudes em relação à massa corporal de escolares de $4^{\mathrm{o}}$ e $5^{\underline{0}}$ anos, de escolas municipais de Itajaí-SC. A prevalência de obesidade abdominal $(11,3 \%)$ na população investigada foi similar àquela observada entre os escolares de dez anos em Pernambuco $(13,8 \%){ }^{20}$

Entre as crianças avaliadas, aquelas em condição socioeconômica mais favorável apresentaram maior prevalência de obesidade abdominal. Fernandes et al. ${ }^{10}$ também observaram maior chance de obesidade abdominal entre os adolescentes com condição socioeconômica alta, e principalmente matriculados na rede privada de ensino, bem como do sobrepeso corporal materno, ou de ambos os pais, associados à obesidade abdominal entre os adolescentes investigados. ${ }^{10}$ Entre adolescentes de cidade com ÍDH considerado médio/baixo, Minatto et al. ${ }^{21}$ observaram padrões mais inadequados de composição corporal, entre aqueles de estrato socioeconômico alto e intermediário.

É importante destacar que os métodos utilizados para mensurar e avaliar a condição socioeconômica nos indivíduos do presente estudo difere daqueles utilizados nos estudos que foram 
comparados. ${ }^{10,21}$ Também é importante considerar a faixa etária dos escolares avaliados, pois a idade variou de 7,7 a 14,3 anos, uma vez que ocorrem modificações importantes na composição corporal da infância para a adolescência. ${ }^{7}$

Evidências da literatura têm apontado que a obesidade geral é um fenômeno de múltiplas origens e substancialmente influenciado por fatores genéticos e ambientais. ${ }^{1,22}$ Todavia, independentemente dos diferentes fatores genéticos ou ambientais associados ao desenvolvimento da obesidade, é consenso na literatura que o núcleo familiar é um agente em potencial associado a esse processo. ${ }^{10}$

Dentre os mecanismos que explicam a maior prevalência de obesidade em crianças e adolescentes de melhor renda, destacam-se: a maior concentração de renda/disponibilidade de alimentos para o jovem em questão (menor número de pessoas na residência e melhor condição econômica) e maior prática de comportamentos sedentários em casa (número de televisores na residência). ${ }^{10}$

Estudos sugerem que as preferências alimentares dos pais influenciam e modulam aquelas de seus filhos. ${ }^{1,23}$ A exposição desde os primeiros anos de vida aos alimentos preferidos pelos pais favorece o desenvolvimento das preferências alimentares da criança. ${ }^{23}$

Dentre as variáveis ligadas à percepção e às atitudes em relação à massa corporal, observouse que crianças com obesidade abdominal apresentaram maior prevalência de perceberem sua massa corporal como acima do ideal, e também tiveram sua massa corporal percebida pelos pais como inadequada mais frequentemente. Além disso, tais crianças apresentaram maiores chances de estarem insatisfeitas com sua imagem corporal, bem como de referirem realização de tentativas para perda de massa corporal.

Pesquisa demonstrou que muitas crianças, principalmente meninas, na idade de cinco anos, estão insatisfeitas com seu corpo, demonstrando preocupações sobre se tornar acima da massa corporal e aderindo a estratégias para perder massa corporal, tais como comer menos e realizar exercícios físicos. ${ }^{24}$ Embora o começo da puberdade aumente o desejo de ser mais magro, há evidências de que no período pré-puberal as crianças também se preocupem com seus corpos e busquem perder massa corporal. ${ }^{25}$

Fatores biológicos, psicológicos e socioculturais têm sido relevantes no entendimento do desenvolvimento de preocupações sobre imagem corporal e estratégias para perda de massa corporal entre crianças, e o principal indicador de utilização de estratégias para perder massa corporal pelas crianças tem sido considerado a pressão dos pais, dos colegas ou da mídia. ${ }^{26} \mathrm{~A}$ percepção dos pais sobre a massa corporal da criança está associada aos níveis de insatisfação corporal e aos comportamentos a ela relacionados em crianças. ${ }^{27}$ 
Ricciardelli \& McCabe, ${ }^{28}$ em estudo de revisão com avaliados compreendidos na faixa etária de seis a 11 anos, observaram que de 20 a 55,6\% das meninas referiram realizar dietas para perder massa corporal; entre os meninos o percentual variou de 31 a 39\%. A prática de exercício para perder massa corporal foi indicada por $43,5 \%$ das meninas e $36,5 \%$ dos meninos. Outras práticas, como vômito autoinduzido, foram observadas em 1,8 e 0,7\% das meninas e meninos, respectivamente. ${ }^{28}$

Entre as razões que levam crianças obesas a buscar ajuda para o problema da insatisfação com excesso de massa corporal, está o incômodo com sua imagem corporal, que pode vir a interferir com seu senso de autoestima. Especialmente num contexto em que o corpo magro, esbelto, é valorizado e as roupas da moda são confeccionadas em tamanhos pequenos, este incômodo parece ser maior e vivenciado com sentimentos de raiva, angústia e culpa. ${ }^{29}$

Desta forma, variáveis tanto dos pais como das crianças estiveram associadas à prevalência de obesidade abdominal entre as crianças avaliadas. Contudo, é importante cautela na interpretação de nossos resultados, em função do delineamento transversal do estudo. Por outro lado, as técnicas utilizadas na seleção de escolas e das crianças, e boa participação das crianças no estudo, indicam que os dados podem ser extrapolados para crianças de $4^{\underline{0}}$ e $5^{\underline{0}}$ anos de escolas municipais da cidade. Além disso, o efeito do delineamento foi considerado nas análises.

Existem discussões em relação ao ponto de corte para discriminar obesidade abdominal em crianças. Os pontos de corte propostos por Taylor et al. ${ }^{16}$ foram baseados em estudo com o uso da absortiometria por duplo feixe de raios (DXA), método que tem sido considerado referência na avaliação da composição corporal. ${ }^{30}$

\section{Conclusões}

Por fim, observou-se prevalência de obesidade abdominal entre as crianças avaliadas, similares àquelas observadas em outros estudos no país. A maior renda dos pais, a presença de excesso de massa corporal, bem como as percepções sobre a adequação da massa corporal da criança, permaneceram associadas à maior prevalência de obesidade abdominal. Além disso, a percepção da criança sobre seu estado nutricional, sua insatisfação em relação à imagem do corpo e referência à realização de tentativas para perder massa corporal também estiveram associadas à obesidade abdominal.

Desta forma, os resultados aqui observados reforçam a importância de realização de atividades multidisciplinares, envolvendo as famílias das crianças, com o intuito de reduzir a prevalência de obesidade abdominal e permitir maior qualidade de vida às crianças. 


\section{Referências}

1. Van Hook J, Altman CE, Balistreri KS. Global patterns in overweight among children and mothers in less developed countries. Public. Health Nutr. 2012; 14:1-9.

2. Aussem A, Tchernof A, Morais SR, Rome S. Analysis of lifestyle and metabolic predictors of visceral obesity with Bayesian Networks. BMC Bioinformatics. 2010;11:487.

3. Garnett SP, Baur LA, Srinivasan S, Lee JW, Cowell CT. Body mass index and waist circumference in midchildhood and adverse cardiovascular disease risk clustering in adolescence. Am. J. Clin. Nutr. 2007; 86(3):549-55.

4. Haas GM, Bertsch T, Schwandt P. Trends of Components of the metabolic syndrome in german first graders throughout 10 years: the pep family heart study. Cholesterol. 2012; 2012:231962.

5. Weiss R, Bremer AA, Lustig RH. What is metabolic syndrome, and why are children getting it? Ann. NY Acad. Sci. 2013; 1281:123-40.

6. Pereira PF, Serrano HMS, Carvalho GQ, Lamounier JA, Peluzio MCG, Franceschini SC, Priore SE. Circunferência da cintura e relação cintura/estatura: úteis para identificar risco metabólico em adolescentes do sexo feminino?. Rev. Paul. Pediatr. 2011; 29(3):372-7.

7. Chrzanowska M, Suder A, Kruszelnicki P. Tracking and risk of abdominal obesity in the adolescence period in children aged 7-15. The cracow longitudinal growth study. Am. J. Hum. Biol. 2012; 24(1):62-7.

8. Kahn HS, Imperatore G, Cheng YJ. A population-based comparison of BMI percentiles and waistto-height ratio for identifying cardiovascular risk in youth. J. Pediatr. 2005; 146(4):482-8.

9. Modi N, Murgasova D, Ruager-Martin R, Thomas EL, Hyde MJ, Gale C, et al. The influence of maternal body mass index on infant adiposity and hepatic lipid content. Pediatr. Res. 2011; 70(3):287-91.

10. Fernandes RA, Casonatto J, Christofaro DGD, Cucato GG, Oliveira ARd, Freitas Júnior IF. Fatores familiares associados à obesidade abdominal entre adolescentes. Rev. Bras. Saúde Mater. Infant. 2009; 9(4):451-457.

11. United Nations. IDH que mede desigualdade aponta queda de 19\% no país. Brasília: PNUD; 2011.

12. Barros F, Victora C. Epidemiologia da saúde infantil: um manual para diagnósticos comunitários. São Paulo: HUCITEC/UNICEF; 1998.

13. Instituto Brasileiro de Geografia e Estatística. Censo demográfico 2000. Agregado por setores censitários dos resultados do universo. Rio de Janeiro: IBGE; 2003. p. 157.

14. Tiggemann M, Wilson-Barrett E. Children's figure ratings: relationship to self-esteem and negative stereotyping. Int. J. Eat. Disord. 1998; 23(1):83-8.

15. Lohman T, Roche A, Martorell R. Anthropometric standardization reference manual. Champaign: Human Kinetics Books; 1988.

16. Taylor RW, Jones IE, Williams SM, Goulding A. Evaluation of waist circumference, waist-to-hip ratio, and the conicity index as screening tools for high trunk fat mass, as measured by dual-energy X-ray absorptiometry, in children aged 3-19 y. Am. J. Clin. Nutr. 2000; 72(2):490-5. 
17. World Health Organization. Growth reference 5-19 years. BMI-for-age (5-19 years). Geneva: WHO; 2010.

18. World Health Organization. Obesity: preventing and managing the global epidemic. Report of a WHO consultation. Geneva: WHO; 2000. World Health Organ. Tech. Rep. Ser; 894.

19. Victora CG, Huttly SR, Fuchs SC, Olinto MT. The role of conceptual frameworks in epidemiological analysis: a hierarchical approach. Int. J. Epidemiol. 1997; 26(1):224-7.

20. Pinto IC, Arruda IK, Diniz Ada S, Cavalcanti AM. Prevalence of overweight and abdominal obesity according to anthropometric parameters and the association with sexual maturation in adolescent schoolchildren. Cad. Saúde Pública. 2010; 26(9):1727-37.

21. Minatto G, Pelegrini A, Silva DAS, Silva AFd, Petroski EL. Composição corporal inadequada em adolescentes: associação com fatores sociodemográficos. Rev. Paul. Pediatr. 2011; 29(4):553-9.

22. Wang Y, Lim H. The global childhood obesity epidemic and the association between socio-economic status and childhood obesity. Int. Rev. Psychiatry. 2012; 24(3):176-88.

23. Birch LL, Fisher JO. Development of eating behaviors among children and adolescents. Pediatrics. 1998; 101(3 Pt 2):539-49.

24. Davison KK, Markey CN, Birch LL. Etiology of body dissatisfaction and weight concerns among 5-year-old girls. Appetite. 2000; 35(2):143-51.

25. Vander Wal JS, Thelen MH. Eating and body image concerns among obese and average-weight children. Addict. Behav. 2000; 25(5):775-8.

26. McCabe MP, Ricciardelli LA. Body image and strategies to lose weight and increase muscle among boys and girls. Health Psychol. 2003; 22(1):39-46.

27. McCabe MP, Ricciardelli LA. A prospective study of pressures from parents, peers, and the media on extreme weight change behaviors among adolescent boys and girls. Behav. Res. Ther. 2005; 43(5):653-68.

28. Ricciardelli LA, McCabe MP. Children's body image concerns and eating disturbance: a review of the literature. Clin. Psychol. Rev. 2001; 21(3):325-44.

29. Carvalho AMP, Cataneo CC, Galindo EMCG, Tomain C. Auto-conceito e imagem corporal em crianças obesas. Paidéia. 2005; 15(30):131-139.

30. Sant'Anna M, Priore S, Franceschini S. Métodos de avaliação da composição corporal em crianças. Rev. Paul. Pediatr. 2009; 27(3):315-321. 
